Cahiers de philosophie de l'université de

\title{
Les attitudes russelliennes
}

Fabien Schang

\section{(2) OpenEdition}

Journals

Édition électronique

URL : https://journals.openedition.org/cpuc/328

DOI : 10.4000/cpuc.328

ISSN : 2677-6529

Éditeur

Presses universitaires de Caen

\section{Édition imprimée}

Date de publication : 31 décembre 2017

Pagination : 149-168

ISBN : 978-2-84133-859-7

ISSN : 1282-6545

Référence électronique

Fabien Schang, "Les attitudes russelliennes », Cahiers de philosophie de l'université de Caen [En ligne], 54 | 2017, mis en ligne le 01 février 2019, consulté le 31 janvier 2023. URL : http:// journals.openedition.org/cpuc/328; DOI : https://doi.org/10.4000/cpuc.328

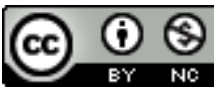

Creative Commons - Attribution - Pas d'Utilisation Commerciale 4.0 International - CC BY-NC 4.0 https://creativecommons.org/licenses/by-nc/4.0/ 


\section{Les attitudes russelliennes ${ }^{1}$}

\section{Logique et croyance}

Russell prétend qu'un examen des croyances est indispensable pour définir nos raisonnements quotidiens et comprendre ce que les philosophes entendent par la notion de vérité:

La vie intellectuelle globale se compose de croyances, ainsi que du passage de la croyance à une autre que l'on appelle «raisonnement». Les croyances apportent la connaissance et l'erreur; elles sont les véhicules de la vérité et de la fausseté. La psychologie, la théorie de la connaissance et la métaphysique gravitent autour de la croyance, et notre approche philosophique dépend en large partie de l'idée que nous nous faisons de la croyance ${ }^{2}$.

Cela étant, l'auteur considère qu'une étude de ces croyances n'a aucun rapport avec la logique, laquelle concerne uniquement le vrai et le faux. En d'autres termes, Russell associe croyance et psychologie tout en réservant le domaine de la logique au thème de la proposition, vraie ou fausse par définition. Une certaine théorie de la vérité sous-tend son rejet primordial d'une logique épistémique; si la croyance a un intérêt philosophique, c'est parce que Russell se sert de son examen pour discerner la théorie de la vérité que défendent plusieurs philosophes: la théorie de la vérité-cohérence est une cible principale du Britannique, qui défend pour sa part une théorie de la vérité-correspondance en caractérisant la proposition par sa relation de correspondance ou de non-correspondance avec un fait.

1. Cet article est extrait d'une sous-section de la thèse de doctorat de philosophie de l'auteur: Philosophie des modalités épistémiques: la logique assertorique revisitée, rédigée à l'université Nancy 2 - Lorraine, sous la direction du professeur Gerhard Heinzmann, 2007.

2. B. Russell, Analysis of Mind, Londres - New York, G. Allen \& Unwin - Macmillan, 1921, «Lecture XII : Belief», p. 231. L'ensemble des traductions de l'anglais vers le français de cet article sont de l'auteur. 
La suite va tenter de montrer que la définition de la croyance telle qu'on la trouve dans la logique épistémique introduite par Hintikka ${ }^{3}$ est inapplicable, voire inconcevable au sein de la philosophie de la logique de Russell; pour ce faire, considérons tout d'abord ce que l'auteur entend par les notions de vérité et de fait. C'est à partir de ces deux notions basiques qu'il définit les notions consécutives de proposition et de croyance.

\section{Modalités et propositions}

L'analogie structurelle entre modalités et quantificateurs a été fréquemment évoquée pour jeter les bases d'une logique des attitudes. Or, il semble que Russell n'était pas sensible à cet argument formel, probablement parce que l'identité de structures ne suffisait pas à ses yeux pour parler de "vérités logiques». La vérité logique présuppose la notion de vérité, mais celle-ci renvoie à une correspondance avec des faits et non à une simple similarité entre des formes d'énoncés. Le terme de vérité n'est pas un marque-place ou une valeur simplement algébrique, ici, et un symbole doit être dans une certaine relation avec un «fait» pour être qualifiable de vrai. On retrouve dans les écrits de Russell la même critique que celle aperçue chez Frege, concernant les modalités aléthiques; en effet, Russell assimile plus ou moins la «nécessité» d'une proposition au sentiment de certitude qu'un énoncé inspire chez le locuteur, et l' "impossibilité" au sentiment de certitude de sa fausseté. Or, ces deux sentiments opposés décrivent un degré optimal de croyance qui, comme nous allons le voir, est strictement dissocié de la logique selon Russell:

Il n'y a pas de notion logique fondamentale de nécessité, ni par conséquent de possibilité. Si cette conclusion est valide, le thème de la modalité devrait être banni de la logique, puisque les propositions sont simplement vraies et fausses ${ }^{4}$.

Les critiques de Russell contre les notions de nécessité et de possibilité visaient principalement le «raisonnement symbolique» de Hugh MacColl, l'un des promoteurs de la logique modale interprétée avec Peirce. Selon MacColl, il n'y a pas deux mais cinq classes de "propositions» (statements) distinctes: une proposition est vraie, fausse, variable (parfois vraie et parfois fausse), certaine, ou impossible. Ces propriétés sémantiques ne s'excluent

3. L'auteur tient à remercier les rapporteurs de cet article pour leurs précieuses corrections et remarques.

4. B. Russell, «Necessity and Possibility» [1905], in Foundations of Logic: 1903-05, A. Urquhart, A. C. Lewis (éd.), Londres - New York, Routledge (The Collected Papers of Bertrand Russell; 4), 1994, p. 507-520, ici p. 520. 
pas mutuellement: une proposition est impossible lorsque sa contradictoire est certaine; si une proposition est certaine, alors elle est vraie également; si elle est impossible, alors elle est fausse également, etc. Une telle distinction sémantique préfigure la distinction actuelle des modalités formelles: $\square \phi$, $\phi, \neg \phi, \nabla \phi \equiv(\diamond \phi \wedge \diamond \neg \phi)$, et $\square \neg \phi$ symbolisent respectivement le fait pour une proposition $\phi$ d'être nécessaire, vraie, fausse, variable, et impossible 5 . Or, Russell reproche à cette distinction de reposer sur une confusion entre deux aspects de la proposition: son expression verbale, que nous appelons l'énoncé, et le contenu même de cette expression, c'est-à-dire le contenu propositionnel ou proposition au sens propre (la pensée frégéenne). Si l'énoncé «la porte est ouverte» est variable plutôt que vrai, c'est parce que sa valeur de vérité peut changer selon le contexte de discours et le locuteur qui le prononce; par opposition, un énoncé tel que « $2+2=4$ » est nécessaire parce qu'il est vrai dans n'importe quel contexte d'énonciation et pour n'importe quel locuteur, et $« 2+2 \neq 4$ » est donc impossible.

La réponse de Russell à cette interprétation modale des «propositions » (ou pseudo-propositions) comporte deux arguments ${ }^{6}$.

Premièrement, l'interprétation modale porte sur des expressions incomplètes, c'est-à-dire des énoncés qui ne restituent pas clairement le contenu de la proposition qu'ils visent à exprimer; dans l'expression «la porte est ouverte», cet énoncé exprime une pensée incomplète et n'est complété que par une formule plus précise du type «la porte est fermée» à l'emplacement $x$ et au temps $t$ '. Dans ce cas, l'énoncé enrichi exprime une pensée complète et n'est plus variable mais soit vrai, soit faux.

Deuxièmement, l'interprétation modale provient selon Russell d'une confusion entre une proposition et une fonction propositionnelle. Une expression telle que « $x$ est un avocat» n'est pas nécessaire parce qu'elle n'est pas vraie pour tout $x$, mais elle est variable parce que vraie pour certains $x$ et fausse pour d'autres; une expression telle que «la porte est fermée au temps $x$ » est variable également, parce qu'elle est vraie pour certains $x$ mais pas pour tout $x$. À partir de cette analyse des modalités de MacColl, Russell affirme sa position classique en maintenant une distinction entre

5. Les lettres de variables propositionnelles grecques $\phi$, $\psi$, etc. symbolisent des propositions de complexité quelconque, atomiques ou moléculaires, tandis que les lettres latines $p, q$, etc. renvoient à des propositions uniquement atomiques. Malgré cette distinction d'usage, l'article conservera les lettres latines dans la mesure où elles apparaissent dans des citations d'auteurs.

6. La critique de Russell se trouve dans son «Review of H. MacColl's Symbolic Logic and Its Applications", Mind, ${ }^{\circ}$ 15, 1906, p. 255-26o. Pour plus de détails sur la logique modale de Hugh MacColl et les objections fondamentales portées par Russell, voir par exemple F. Schang, «MacColl's Modes of Modalities», Philosophia Scientioe, vol. 15, n 1, 2011, p. 163-188. 
l'expression d'une proposition et le contenu d'une proposition : parce que la logique des propositions s'intéresse uniquement au contenu de ces propositions, lesquelles doivent être strictement distinguées de leur moyen d'expression qu'est l'énoncé, une proposition est soit vraie soit fausse et le mode de vérité ne concerne pas la logique en tant que telle. Et puisque la certitude représente la version épistémique de la nécessité (est certain ce qu'un sujet considère comme nécessairement vrai), une logique épistémique est légitime pour MacColl mais ne peut l'être pour Russell.

\section{Croyances et propositions}

Russell a exposé notamment sa définition de la croyance dans deux articles ${ }^{7}$, où deux questions cruciales sont posées: qu'est-ce qui distingue les attitudes propositionnelles (fonctions de signification) des fonctions de vérité, d'une part; que faut-il comprendre par l'expression "A croit $p$ ", d'autre part. Russell avait bien conscience des difficultés créées en logique par les attitudes propositionnelles: ce ne sont pas des fonctions de vérité, puisque la croyance à une proposition quelconque $\phi$ (symbolisée par $f \phi$ ) n'implique pas la croyance à toutes les propositions $\psi$ équivalentes à $\phi$. Si l'on définit la relation logique d'équivalence comme une relation d'identité de valeurs de vérité: $(\phi \equiv \psi) \Leftrightarrow(f \phi \equiv f \psi)$, celle-ci ne permet donc pas d'identifier des croyances. Ainsi, je peux croire une certaine proposition vraie (que Paris est la capitale de la France, par exemple) sans que cela m'oblige à croire toute autre proposition de même valeur de vérité (que Minsk est la capitale de la Biélorussie, entre autres). Une telle attitude serait absurde, bien sûr; elle devrait pourtant s'imposer à la fonction de croyance en vertu de la loi de vérifonctionnalité, selon laquelle toute fonction logique appliquée aux mêmes valeurs de vérité produit le même effet. Russell en conclut qu'une fonction linguistique n'est pas toujours une fonction de vérité: toute fonction de vérité est une fonction de signification, puisqu'elle contribue à la signification des propositions, mais la réciproque n'est pas toujours vraie. La question est de savoir ce qui distingue le fait d'être vrai du fait d'être significatif, ou pourvu de sens. Pour expliquer l'irréductibilité des attitudes propositionnelles à des fonctions de vérité, Russell déclare que la proposition ne remplit pas un rôle logique dans une attitude:

7. B. Russell, «Truth-Functions and Meaning-Functions» [1923], in Essays on Language, Mind and Matter: 1919-26, J. G. Slater, B. Frohmann (éd.), Londres - New York, Routledge (The Collected Papers of Bertrand Russell; 9), 1994, p. 156-158; B. Russell, «What is Meant by "A believes p" ?" [1923], in Essays on Language, Mind..., p. 159. 
Il y a quelque chose à dire en faveur de l'idée selon laquelle les propositions peuvent se présenter de deux manières, (a) comme des assertions, (b) comme des faits, et selon laquelle les déclarations (statements) dans lesquelles elles se présentent comme des assertions sont toujours des fonctions de vérité, bien que celles dans lesquelles elles se présentent comme des faits peuvent ne pas l'être. Une telle théorie exigera une analyse logique de ce que l'on entend par «déclaration portant sur un fait»; et aussi de ce que l'on entend, en logique, par «assertion ${ }^{8}$.

Russell distingue ici l'assertion de la déclaration (statement), c'est-à-dire la «pseudo-proposition» de MacColl, parce qu'une déclaration peut être fausse alors que Russell conçoit l'assertion comme une déclaration vraie par définition. Le même problème s'était posé dans le Begriffsschrift de Frege, au sujet de la notion de jugement comme "reconnaissance de la vérité». Lorsqu'on la considère comme un simple acte de discours, l'assertion peut être assimilée en revanche au jugement de Frege et traitée comme synonyme de la simple déclaration.

Que faut-il entendre par un «fait», et pourquoi une proposition n'estelle plus une fonction de vérité lorsque l'on s'intéresse à elle en tant que fait et non en tant qu'assertion? Tout d'abord, Russell entend par «fait» la réalisation d'un événement quelconque. Une croyance est un fait, un fait psychologique. Une déclaration, c'est-à-dire la production d'un énoncé, est un fait, un fait linguistique.

Ainsi s'en explique Russell:

Qu'entend-on par "A croit $p$ »? Il y a une manifestation dans A, appelée «croyance». C'est un fait comme un autre ${ }^{9}$.

Mais lorsqu'une fonction de signification est une fonction de vérité, la proposition à laquelle est attribuée une valeur de vérité ne représente pas un fait quelconque. Dans l'énoncé "Platon est grec», par exemple, l'individu nommé Platon est un constituant de la proposition qui dit que Platon est grec, et le fait que Platon est grec (la pensée frégéenne) est exprimé par cet énoncé; mais deux types de faits distincts sont produits lors d'une énonciation: le fait contenu dans l'énoncé, c'est-à-dire la proposition frégéenne, et le fait d'énoncer ce contenu. Le second fait, celui d'avoir exprimé la proposition que Platon est grec par le biais de l'énoncé "Platon est grec», est d'ordre linguistique et concerne notre discours sur les choses; le premier fait, le fait que Platon est grec, est

8. B. Russell, «Truth-Functions and Meaning-Functions», p. 156.

9. B. Russell, «What is Meant by “A believes $p$ ”?», p. 159. 
d'ordre ontologique et concerne les choses elles-mêmes. Russell explique ainsi que, dans une fonction de vérité, la proposition n'est pas considérée comme le fait linguistique qu'est l'acte d'énoncer. Elle n'est pas considérée non plus comme le symbole du fait qui rend la proposition vraie ou fausse, car réduire la proposition à un symbole impliquerait que deux expressions issues de deux langues naturelles distinctes ne représentent pas la même proposition: si «Platon est grec» et «Plato is Greek» n'exprimaient pas la même proposition, alors ces expressions ne seraient pas vraies ou fausses conjointement. Et puisque, dans une fonction de vérité $f \phi$, la proposition $\phi$ est le contenu propositionnel de l'énoncé, à savoir ce qui est rendu vrai ou faux par la réalisation d'un fait ontologique, la proposition $\phi$ ne dépend pas du langage dans lequel elle est exprimée mais est un constituant du fait qui rend $f \phi$ vraie ou fausse. Seules les propositions sont des fonctions de vérité, autrement dit, et les énoncés qui les expriment sont des fonctions de signification de ces propositions.

Contrairement aux fonctions de vérité, dont les constituants sont en relation avec ce qui les rend vrai ou faux (les états de choses ou faits ontologiques), la croyance porte sur un fait psychologique parce que ses constituants ne sont pas définis en termes de ce qui les rend vrais ou faux. L'ambiguïté vient de la notion de fait, qui peut être d'ordre social, psychologique, linguistique, ou ontologique; un fait peut être logique ou psychologique, selon Russell, mais seul le fait exprimant une relation de correspondance avec la réalité peut être qualifié de vrai ou de faux. La croyance n'est pas évacuée pour autant de l'analyse des fonctions de vérité et de leurs propositions, mais elle correspond à ce que Russell appelle une fonction de signification et n'équivaut pas à une fonction de vérité: une croyance est un fait psychologique qui porte sur un contenu propositionnel vrai ou faux, mais la vérité ou fausseté de ce contenu (de la proposition frégéenne) ne dépend pas de la croyance, qui est un fait strictement psychologique. Une croyance se manifeste dans des circonstances diverses: si le sujet croyant a une raison de considérer quelque chose comme vrai, ou s'il dispose d'une preuve en faveur de cette vérité. Mais on voit bien ici que les circonstances d'une croyance ne déterminent pas elles-mêmes ce qui est vrai ou faux dans le monde. Pour cette raison, Russell déclare que

la logique ignore par convention ces circonstances, toutefois; elle suppose que, pour toute croyance donnée, il y a un certain fait défini qui la rend vraie (ou fausse) ${ }^{10}$.

10. B. Russell, «Truth-Functions and Meaning-Functions», p. 157. 
À partir de cette distinction entre croyance et vérité, on peut redéfinir la notion de proposition et compléter ce que nous avions dit plus tôt au sujet de la relation entre proposition et énoncé: la proposition désigne la classe de toutes les croyances ou de leurs expressions linguistiques qui sont rendues vraies ou fausses par un fait.

Une proposition est donc une classe de faits, psychologiques ou linguistiques, définis comme ceux qui ont une certaine relation (qui peut être assertion ou négation (denial), selon les cas) à un certain fait ${ }^{11}$.

Cette définition de la proposition évite le recours aux entités abstraites que l'on trouvait chez Frege, les pensées, et caractérise la proposition comme une classe de faits linguistiques rendus vrais par un même fait ontologique ou état de choses; et puisque ces faits linguistiques expriment la totalité des croyances qui caractérisent une proposition, ce sont donc ce que nous appelons des énoncés ou phrases déclaratives. Lors de l'expression d'une croyance par le biais d'un énoncé, une pensée commune est supposée «saisie» par plusieurs sujets croyants et se manifeste sous la forme linguistique d'un ou plusieurs énoncés; mais l'entité idéale que représente la proposition n'est définie chez Russell que de manière indirecte, par le biais de la classe de tous les énoncés qui l'expriment. Ce n'est donc pas la proposition en tant que telle qui est exprimée dans une croyance mais seulement un membre de cette proposition, et ce membre qu'est l'énoncé appartient à une classe de croyances (ou de leurs expressions symboliques). On retrouve ici la même distinction que celle aperçue chez Frege entre une pensée et l'entité linguistique qui sert à l'exprimer; dans les deux cas, la pensée est une entité extralinguistique qui s'exprime à travers un énoncé.

Récapitulons: une croyance est un fait psychologique; la vérité est une relation entre une proposition et un fait ontologique, indépendant du sujet croyant. Les croyances sont exprimées par le biais d'expressions symboliques, et celles-ci expriment une proposition. Plusieurs énoncés sont reliés à une seule et même proposition lorsqu'ils parlent du même fait ontologique, celui qui les rend tous vrais ou faux. Lorsque l'énonciation, c'est-à-dire l'acte d'exprimer une proposition par le biais d'un énoncé, est considérée comme un fait à part entière, la vérité ou fausseté n'est plus en cause et la logique n'est donc plus concernée puisque celle-ci s'intéresse uniquement aux conditions de vérité et de fausseté des propositions. La distinction établie par Russell entre attitudes épistémiques et fonctions de vérité réside dans une distinction de la nature des faits qui les concernent, psychologiques ou linguistiques pour les premières, ontologiques pour les secondes:

11. Ibid. 
Une fonction de vérité de $p$ est rendue vraie (ou fausse) par le même fait qui rend $p$ vraie (ou fausse); en vertu de la définition ci-dessus de la proposition, c'est donc la même proposition que $p$, ou la même proposition que non- $p$. De même que deux fonctions de vérité de plusieurs arguments sont identiques si elles sont équivalentes [...]. Lorsqu'une croyance est vraie, si $f$ est le fait qui la rend vraie nous disons que la croyance a la relation $\mathrm{A}$ (assertion) à $f$. Lorsqu'une croyance est fausse, appelons $\mathrm{N}$ (négation) la relation ${ }^{12}$.

L'assertion telle que Russell l'entend présuppose donc bien la vérité de la déclaration, contrairement à la notion de jugement chez Frege où un jugement asserté peut être faux. Si l'on reprend la distinction de Hanson entre déclarations de fait (toujours vraies) et déclarations factuelles (falsifiables) ${ }^{13}$, l'assertion de Russell s'exprime par le biais d'une déclaration de fait.

Comme indiqué plus haut, l'analyse de la proposition est d'ordre logique lorsqu'elle est considérée du point de vue de son assertion: c'est le cas d'une proposition rendue vraie par un fait; elle n'est plus d'ordre logique lorsque la proposition est considérée du point de vue de son expression: c'est le cas d'un énoncé produit pour exprimer cette proposition. Dans ce dernier cas, on parle d'une proposition au sens de la proposition grammaticale ou énoncé, qui est l'expression d'une croyance. Mais ce sens courant de la «proposition» n'est pas celui qui intéresse Frege et Russell : lorsque nous parlons ici de "proposition", nous faisons allusion uniquement à la proposition logique, c'est-à-dire au contenu d'une proposition grammaticale. Lorsque l'on parle d'un «contenu propositionnel», l'expression «propositionnel» renvoie à la proposition au sens grammatical du terme et le contenu propositionnel correspond à la proposition au sens logique du terme. À cet égard, l'erreur du logicien épistémique serait de confondre les deux types de cas et de parler indifféremment de vérité lorsque l'on dit que la manifestation d'une croyance «correspond» à un fait (psychologique).

Pour définir la forme d'une proposition et la forme d'une croyance C, Russell utilise un symbolisme ensembliste constitué du fait $f$, de l'assertion $\mathrm{A}^{\rightarrow}$ (rendre vrai) et de la négation $\mathrm{N}^{\rightarrow}$ (rendre faux) ${ }^{14}$. On obtient les explications suivantes:

Proposition

$\phi={ }_{\mathrm{df}} \mathrm{A}$ !'f ou N!'f, pour un certain $f$

12. B. Russell, «Truth-Functions and Meaning-Functions», p. 158.

13. N. R. Hanson, "A Note on Statements of Fact», Analysis, vol. 13, nº 1, 1952, p. 24.

14. Voir B. Russell, «Truth-Functions and Meaning-Functions». 


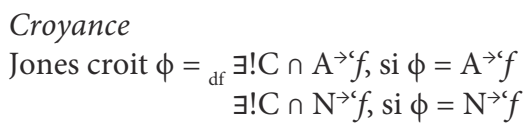

Russell précise sa définition de la proposition en termes de classes de croyances (et de leurs expressions linguistiques) $\mathrm{C}$ rendues vraies ou fausses par un fait, remplaçant les prédicats d'assertion $\mathrm{A}$ et de négation $\mathrm{N}$ par ceux de vérification $\mathrm{V}$ et de falsification $\mathrm{F}^{15}$. Quoi qu'il en soit, la proposition est dans les deux cas une classe de croyances dont l'appartenance au même ensemble est déterminée par un fait ontologique commun.

Pour revenir sur une question précédente: comment établir l'identité de deux croyances, notons que la réponse de Russell ne portera évidemment pas sur ce qui les distingue en tant que faits psychologiques ou linguistiques - le «fait propositionnel» ne compte pas, puisque l'identité est liée à la notion de vérité et se rapporte ainsi au fait qui rend vraie une proposition. Deux croyances sont identiques si leur expression linguistique porte sur la même proposition, donc si elles appartiennent toutes deux à la classe des énoncés qui sont rendus vrais ou faux par le même fait. À noter que l'expression «croyance vraie» signifie de manière elliptique que son énoncé est rendu vrai par le fait qu'il exprime: comme le dit Russell, la vérité est « une qualité entièrement dépendante du rapport existant entre la croyance et les objets extérieurs $»^{16}$.

Il y a une conséquence essentielle dans cette organisation entre les notions de logique et de psychologie, distinguées par le critère de vérité: tout fait n'est pas constitutif de la vérité, et seul un certain type de fait peut être qualifié ainsi. C'est le fait logique, et ce fait est indissociable du fait ontologique dans la mesure où il porte sur la relation de correspondance ou non-correspondance entre une proposition, exprimée par une classe d'énoncés, et ce que cette proposition dénote. Le fait que Pierre croie que Platon est grec n'est pas «vrai» en tant que tel: le fait que Pierre produise ici ce qui s'avère être une assertion (une proposition vraie) est un fait linguistique portant sur un énoncé mais pas sur ce qui rend cet énoncé vrai ou faux; pour cette raison, le fait d'énoncer quelque chose qui s'avère vrai par ailleurs (le fait de l'asserter) est psychologique et n'a pas de valeur de vérité propre.

La distinction établie par Russell entre un fait primaire (logique) et un fait secondaire (non-logique) préfigure en un sens le théorème d'introspection du système modal $\mathrm{S}_{4}$ : si un agent sait qu'une proposition

15. Voir B. Russell, «What is Meant by "A believes $p$ ”?».

16. B. Russell, Problèmes de philosophie, S.-M. Guillemin (trad.), Paris, Payot (Petite bibliothèque Payot; 79), 1965, «Le vrai et le faux», p. 144. 
est vraie, alors il sait qu'il sait qu'elle est vraie. Pour distinguer les deux types de faits, Russell recourt en effet à une forme d'itération:

Ainsi la proposition qui apparaît lorsque nous assertons une proposition est tout à fait différente de celle qui apparaît lorsque nous assertons que nous assertons une proposition; celle-ci est un fait psychologique ou linguistique, celle-là une classe de ces faits ${ }^{17}$.

Mais il ne faut pas confondre l'itération des modalités épistémiques et l'itération des assertions décrite ci-dessus; l'assertion correspond chez Russell à ce que lui-même entend par une assertion logique : ce n'est pas un acte d'assertion, comparable à ce que décrit la barre de jugement de Frege, mais un fait logique relatif à la correspondance entre une proposition et un fait qui la rend vraie. Asserter que l'on asserte quelque chose décrit en revanche un acte commis par le locuteur: dans ce cas, Pierre dit que l'énoncé qu'il exprime se rapporte à une proposition vraie, et la croyance devient alors un constituant du fait psychologique qu'est l'énonciation. L'itération de la croyance change ainsi le statut de l'assertion qui, lorsqu'elle est redoublée, ne porte plus sur un fait logique mais sur un fait psychologique. On retrouve ici l'idée du changement de référence de Frege dans un contexte de discours indirect, lorsque l'énoncé n'est plus considéré pour sa valeur de vérité mais pour sa valeur cognitive, ou informative. Russell distingue clairement cette valeur du domaine des faits logiques, limités pour leur part à l'étude de la relation entre langage et monde.

L'analyse logique de Russell rend donc absurde le projet même de logique épistémique, à moins de confondre les notions d'être vrai et d'être le cas. Un fait psychologique peut être le cas, mais un seul type de cas intéresse le Russell logicien, dont la distinction entre différents ordres de faits interdit toute confusion entre plusieurs genres de vérité. Cette acception précise de la notion de vérité a au moins deux conséquences pour la sémantique modale.

Revenons dans l'ordre sur ce qui distingue une proposition et une attitude propositionnelle, puis sur la notion de vérité selon Russell.

\section{Propositions et attitudes propositionnelles}

L'explication de Russell semble confirmer l'approche nominaliste des "propositions" au sein d'ensembles épistémiques qui caractérisent un modèle logique: lorsque la logique modale épistémique définit une

17. B. Russell, «Truth-Functions and Meaning-Functions», p. 158. 
croyance comme un ensemble de formules, ces formules représentent dans un langage formel des énoncés des langues naturelles et non des propositions telles que Russell les a définies. Dans la mesure où ces énoncés expriment un ensemble de croyances communes au sein d'un langage, ce n'est donc pas un ensemble de "propositions » qui définit la croyance; bien au contraire, comme l'explique Russell:

Lorsque nous considérons une croyance, la proposition au sens qui vient d'être défini ne se présente pas dans notre croyance; ce qui se présente, c'est un membre de la classe qu'est la proposition. Même chose lorsque nous utilisons des mots pour asserter une proposition ${ }^{18}$.

La définition russellienne de la proposition ressemble en un sens à la définition de l'opérateur modal épistémique: de même que la proposition a été interprétée plus haut comme une classe de faits psychologiques (de croyances) ou linguistiques (les énoncés qui les expriment), la croyance de $\phi$ est interprétée en logique modale épistémique comme la classe des mondes possibles où $\phi$ est vraie. Dans les deux cas, l'expression à définir est donc présentée en termes de classes. Mais contrairement à la croyance de Russell, où ce fait psychologique est corrélé à un fait linguistique qui est son énoncé, la croyance se définit en termes de classe de formules chez Hintikka ${ }^{19}$. D'une part, cette classe de formules n'est pas déterminée par le fait qui les rend vraies. On parle de classes aussi bien chez Russell que chez Hintikka, mais le contenu de ces classes est donc distinct. D'autre part, la réduction nominaliste des mondes possibles à des ensembles modèles crée une autre difficulté: une croyance porte sur une formule; mais cette formule représente-t-elle une proposition, un énoncé, ou autre chose? Dans l'esprit de Russell (et Frege), une croyance est exprimée par un énoncé mais désigne une proposition; or si, dans une formule modale de type $\mathrm{B} \phi$, l'objet $\phi$ de la croyance B est exprimé par un énoncé, cet énoncé n'est pas vrai ou faux à proprement parler mais exprime quelque chose, une proposition qui, elle, est rendue vraie ou fausse par un fait (ontologique). Un énoncé n'est donc vrai ou faux que par l'entremise de la proposition qu'il exprime, et une croyance vraie signifie uniquement une croyance dont le contenu - ou idée - exprimé par un énoncé correspond à un fait. Russell suggère la notion d'idée pour exprimer le contenu d'une croyance qui n'est pas une proposition:

18. Ibid., p. 157 .

19. Voir J. Hintikka, Knowledge and Belief: An Introduction to the Logic of the Two Notions, Ithaca, Cornell University Press, 1962. 
Prenez « $\mathrm{A}$ croit que $\mathrm{B}$ a tué $\mathrm{C}$ ». Cela dit que dans $\mathrm{A}$ il y a des manifestations appelées «idées» de $\mathrm{B}$, de tuer et de $\mathrm{C}$, liées d'une certaine manière. Ici la proposition n'apparaît pas, et «A croit $p$ » n'est pas une fonction de $p^{20}$.

On notera que, dans la formulation anglaise de l'attitude propositionnelle, il est écrit «A croit $p$ » $(A$ believes $\mathrm{p})$ et non «A croit que $p$ » $(A$ believes that $\mathrm{p}$ ): l'absence de la forme complétive (that-clause) est une indication grammaticale du fait que le contenu de la croyance n'est pas une proposition, signalée en général par la forme «que $p$ ». Ce détail importe pour éviter la confusion possible entre nos expressions courantes et la forme symbolique appropriée de la croyance.

Une lecture de la sémantique relationnelle (ou modale) en termes russelliens conduirait donc à une impasse: si les constituants d'un ensemble modèle étaient des énoncés, on ne pourrait pas les qualifier eux-mêmes de vrais ou faux sans commettre une confusion des genres; si ces constituants étaient des propositions, il n'y aurait plus seulement un mais plusieurs faits ontologiques à prendre en compte pour évaluer une seule et même croyance dans la mesure où à chaque fait ontologique correspond une proposition unique. On aboutirait à une absurdité dans les deux cas, et ce pour une raison claire: chez Russell, la croyance sert à définir la proposition mais n'est pas elle-même définissable. Une croyance correspond tout au plus à un énoncé, et une classe de croyances constitue une proposition; mais jamais la croyance n'apparaît chez Russell comme un terme à définir, contrairement à la logique modale épistémique. Les classes dont parlent Russell et Hintikka ne sont pas de même nature, encore une fois : elles permettent d'identifier une proposition chez Russell et une attitude propositionnelle chez Hintikka, de sorte que le point de vue que l'un examine est diamétralement opposé au point de vue qui intéresse l'autre. Dans une attitude propositionnelle, la classe à laquelle il est fait allusion détermine l'ensemble des formules que le sujet est disposé à croire; dans une proposition, la classe en question détermine l'ensemble des croyances qui constituent une proposition objective. En d'autres termes, Russell et Hintikka s'expriment en termes ensemblistes mais sur des sujets distincts: le premier s'occupe du contenu des attitudes propositionnelles, le second des attitudes elles-mêmes. Mais aucune circularité ne ressort en réalité des définitions de l'attitude propositionnelle et de la proposition. Certes, la proposition se définit comme un ensemble d'attitudes de croyance chez Russell; mais l'attitude de croyance ne se définit pas comme un ensemble de propositions chez Hintikka. Certes, à une croyance correspond un

20. B. Russell, «What is Meant by "A believes p"?», p. 159. 
énoncé chez Russell tandis qu'à une croyance correspondent plusieurs formules (des énoncés, dirons-nous) chez Hintikka; mais la relation entre croyance et énoncé n'est ambiguë que si l'on confond les points de vue des deux auteurs: une croyance correspond à un énoncé lorsqu'il s'agit de définir la proposition, et une croyance correspond à plusieurs énoncés lorsqu'il s'agit de définir l'attitude d'un sujet à l'égard d'une proposition.

\section{Le concept russellien de vérité}

Que veut-on dire lorsque l'on parle de la «vérité» d'une croyance: qu'une croyance a été effectivement tenue par quelqu'un, ou que le contenu de cette croyance est vrai? La distinction entre ces deux acceptions de la notion de vérité correspond à la distinction entre la vérité d'une attitude propositionnelle et la vérité de la proposition, c'est-à-dire du contenu de l'attitude. La distinction marquée par Russell entre logique et psychologie consiste à restreindre l'application de la notion de vérité au second cas: seules les propositions sont vraies, et une croyance n'est vraie que de manière indirecte (c'est son contenu qui est vrai ou faux).

Mais la situation se complique lorsque l'on entre en logique modale et que l'on aborde la notion de vérité du point de vue ontologiquement neutre de la théorie des modèles. Étant donné son caractère nominaliste, la sémantique relationnelle de Hintikka n'implique évidemment pas l'existence de faits (ontologiques) multiples pour une seule et même croyance et l'énoncé qui lui correspond. Mais dans le même temps, Hintikka insiste à dire que sa sémantique doit exprimer un lien fondamental entre le langage et la réalité. Contrairement à notre recommandation ci-dessus, consistant à ne parler de vrai et de faux que relativement à des états de choses (des faits ontologiques), la vérité telle que l'entend le logicien modal peut être comprise comme un simple fait d'être le cas, sans précision sur le type de cas en vue (social, psychologique, linguistique, ontologique): la vérité signifiera tantôt l'appartenance d'une formule à un modèle, tantôt la correspondance d'une proposition à ce modèle singulier qu'est le monde réel (le seul conçu par Russell). La première forme de vérité désigne une sorte de vérité-cohérence, par opposition à la vérité-correspondance de Russell. Cette différence d'usage de la notion de vérité peut expliquer en grande partie les réticences de ce dernier vis-à-vis de la logique modale, lorsque l'usage de la notion de vérité devient plus libéral. La distinction entre attitudes propositionnelles et propositions est sans réserve, chez Russell:

[dans un énoncé de croyance,] la référence au fait, lequel confère la vérité ou la fausseté, est hors de propos. Une croyance (et son expression linguistique) 
n'est intéressante pour la logique qu'en qualité de véhicule de la vérité ou de la fausseté. Lorsque j'asserte que Socrate est mortel, la manifestation est différente de ce qu'elle est lorsqu'un autre individu asserte la "même» proposition; mais cette différence est sans importance pour la logique, qui ignore tout ce qui concerne l'assertion à l'exception de ce qu'elle a en commun avec d'autres assertions de la «même» proposition ${ }^{21}$.

Pour distinguer ce qui est vrai de ce qui ne peut pas l'être, le symbolisme doit donc être défini en plusieurs éléments distincts. Dans un ensemble de formules indistinctes, chacune de ces formules est distinguée selon sa fonction. On entend par phrase l'inscription matérielle d'une suite de signes; deux phrases distinctes peuvent renvoyer à un même énoncé, et une phrase est identifiée par sa production dans un cas particulier. Par exemple: la phrase «Platon est grec» et cette phrase-ci, «Platon est grec», sont deux phrases (occurrences matérielles) distinctes mais qui produisent un même énoncé. Quant à l'énoncé, nous avons dit depuis le début qu'il correspond à une phrase déclarative exprimant une certaine signification dans une certaine langue; la signification de l'énoncé se définit en termes de vérité et de fausseté, mais un énoncé appartient à une langue naturelle particulière. La vérité ou fausseté de deux énoncés distincts dépend enfin de la proposition qu'ils expriment tous les deux, mais par le biais d'expressions différentes. Deux énoncés peuvent renvoyer à la même proposition, mais ils se distinguent selon qu'ils appartiennent à des langues différentes ou prennent des expressions différentes dans une même langue. Deux exemples: "Platon est grec» est un énoncé de la langue française et "Plato is Greek» est un énoncé de la langue anglaise, mais tous deux expriment la même proposition. Partant de la proposition comme signification hypostasiée et commune à plusieurs énoncés, on peut reformuler la notion de proposition en disant qu'elle correspond chez Russell à une classe d'énoncés, lesquels sont rendus vrais ou faux par un même fait ontologique. Par extension, une fonction de vérité $f \phi$ est une fonction dans laquelle la formule $\phi$ symbolise une proposition, et non un énoncé: la valeur sémantique de $f \phi$ ne change pas selon l'expression choisie pour l'énoncé de $\phi$. Quant à l'assertion, le signe de la barre de jugement de Frege indique le cas où l'assertion est elle-même prise pour un fait. Il faut distinguer alors entre l'assertion $\phi$, qui intéresse la logique, et l'acte d'asserter $\phi$, exprimé par l'assertion d'un énoncé et qui n'intéresse pas la logique mais la psychologie. D’où cette explication de Russell:

21. B. Russell, «Truth-Functions and Meaning-Functions», p. 158. 
Qu'entend-on par « $\vdash p$ »? Ce qui se produit, c'est que j'asserte quelque chose; ce qui se produit, c'est ce dont on asserte la production lorsque j'asserte "A croit $p$ ». Mais la logique n'est pas concernée par ce qui se produit lorsque j'asserte; elle n'est concernée que par ce qui est asserté22.

Et cependant, la suite du passage qui vient d'être cité semble produire une contradiction dans le discours de Russell: "C'est là où une proposition diffère de celle qui est une classe de croyances, que l'on peut qualifier de proposition psychologique. Une proposition logique fait partie d'une croyance individuelle. En assertant " $p$ ou $q$ ", la proposition logique fait partie de la croyance». N'est-ce pas plutôt le contraire, à savoir qu'une proposition psychologique désigne une croyance exprimée dans un énoncé « $p$ », lequel est un élément de la classe propositionnelle «que $p$ »? Jusqu'ici, une proposition n'est pas contenue dans une croyance puisqu'elle est la classe dont les croyances sont les éléments. À défaut de réponse précise sur ce passage ambigu, posons (en vertu de tout ce qui précède) qu'une croyance est exprimée par une proposition psychologique; en ce sens, la proposition psychologique correspond à notre énoncé. Quant à la proposition logique, elle est une classe de propositions psychologiques.

Russell ajoute ainsi une distinction supplémentaire pour évoquer les expressions linguistiques caractérisant soit une croyance à un fait, soit un fait: la proposition logique, qui désigne ce qui renvoie à un fait; la proposition psychologique, qui désigne ce qui renvoie à une déclaration de fait, ou énoncé.

On peut résumer les relations d'ensemble entre énoncé, proposition, valeur de vérité et faits de la façon suivante:

- croyances et énoncés se rapportent au domaine de la psychologie: une croyance s'exprime par le biais d'un certain nombre d'énoncés $\phi_{1}, \phi_{2} \ldots \phi_{n}$;

- propositions et faits se rapportent au domaine de la logique: la proposition que $\phi$ est vraie, si elle exprime le fait ontologique que $\phi$ est le cas; elle est fausse, en cas d'absence d'un tel fait;

- les propositions sont des classes de croyances qui sont rendues vraies par un même fait; la croyance n'a de valeur logique que pour autant qu'elle contribue à exprimer une proposition.

La vérité est décrite ici comme la relation de correspondance entre une proposition ( «que $\phi »$ ) et un fait (noté « $\phi »)$, et la fausseté comme une absence de fait correspondant. Mais à strictement parler, « $\phi »$ n'est qu'un

22. B. Russell, «What is Meant by "A believes p"?», p. 159. 
signe linguistique et ne représente donc pas un fait mais une expression de ce fait: c'est ce que Tarski affirmera en substance dans sa définition de la vérité comme relation entre deux niveaux de langage (le langage-objet et le métalangage) et non entre le langage et la réalité.

$\mathrm{Au}$ total, Russell exige pour la vérité une séparation rigoureuse entre logique et psychologie, la première concernant uniquement la relation entre une proposition et un fait ontologique:

Donc bien que, lorsque j'asserte une proposition, j'asserte nécessairement un membre de la classe qu'est la proposition, en tant que logicien j'ignore en revanche tout ce qui concerne la distinction de ce membre avec les autres. C'est ce qui rend la logique différente de la psychologie, et davantage concernée par la vérité ou la fausseté des croyances ${ }^{23}$.

Quant aux formules de la logique épistémique, elles symbolisent ce que Russell appellerait des "propositions psychologiques» et qui sont exprimées par des énoncés. Les expressions modales $\mathrm{K} \phi$ ou $\mathrm{B} \phi$ sont liées à un fait qui les rend vraies ou fausses, mais elles ne sont pas elles-mêmes vraies ou fausses au sens donné par Russell à ces valeurs de vérité: si je crois que deux et deux font cinq, il est «vrai» de dire que je crois une proposition fausse; mais une vérité psychologique de ce genre est ce que Russell a qualifié plus tôt d' "idée » et qu'il distingue soigneusement des propositions de la logique. Si, en revanche, les faits exprimés par des assertions (ou négations d'assertions) sont des faits d'ordre psychologique, de quoi parle le logicien épistémique si son analyse ne porte pas sur des faits logiques? Pour défendre ce dernier, on répondra que le critère de cohérence des ensembles modèles est une formulation du principe de contradiction et que celui-ci est une proposition logique pour Russell. Or, si les formules modales de croyance sont caractérisées par des principes logiques, pourquoi Russell les maintient-il à l'écart de toute analyse d'ordre logique?

Le fait que Russell assimile les croyances à des faits psychologiques vient de ce que ces derniers ne se définissent pas en termes de vérité et de fausseté, contrairement aux faits logiques: la production des croyances serait due à des faits accidentels qui ne concernent pas les états de choses eux-mêmes, les faits ontologiques. Or, la logique épistémique est une sorte de prolongement de la théorie de l'image tableau de Wittgenstein : le logicien épistémique, contrairement au logicien tel que le conçoit Russell, n' «ignore pas tout» des relations entre les croyances qui sont des membres d'une

23. B. Russell, «Truth-Functions and Meaning-Functions», p. 158. 
classe propositionnelle. Elles peuvent être décrites à la rigueur comme des faits psychologiques, mais elles n'en sont pas moins organisées selon un critère minimal de cohérence logique.

On peut parler à cet égard d'une réduction logiciste des croyances à des ensembles de propositions non contradictoires, dans une logique épistémique telle que celle inaugurée par Hintikka ${ }^{24}$.

\section{Vérité et cohérence}

Une dernière question concerne la relation entre cohérence et vérité: peut-on parler de la cohérence (ou non-contradiction) d'un ensemble d'énoncés sans jamais parler de correspondance avec des faits ontologiques que ces énoncés exprimeraient? Autrement dit: les faits logiques sont-ils parfaitement indépendants des faits ontologiques? Pas pour Russell, qui prétend que les partisans de la théorie de la vérité-cohérence présupposent cela même qu'ils cherchent à éliminer :

en réalité, «cohérence» présuppose la vérité des lois de la logique [...], les lois de la logique fournissent le cadre où vient s'insérer le test de la cohérence, et ces lois mêmes ne peuvent être établies grâce à ce même test [...]. Nous voilà donc ramenés à la correspondance avec les faits, pour définir la nature de la véritée 25 .

Considéré comme un cas limite de la vérité, le critère de cohérence entre des énoncés reposerait donc sur leur relation de correspondance avec des faits. Deux énoncés $\phi$ et $\neg \phi$ sont mutuellement incompatibles au sens où ils ne peuvent pas correspondre à un même fait ${ }^{26}$, de sorte que la structure des faits de langage dépend fondamentalement de la structure des faits du monde. Pour cette raison, distinguer la logique de Russell et la logique de Hintikka comme désignant respectivement une logique ontologique et une logique épistémique ne serait pas une chose correcte: la logique épistémique conserve une base ontologique pour déterminer ses règles de cohérence. On ne peut pas caractériser non plus la logique de Russell comme l'expression d'une théorie de la vérité-correspondance, par opposition à la logique de Hintikka qui serait l'expression d'une théorie de la vérité-cohérence: la différence entre leurs sémantiques ne repose pas sur une différence dans

24. Voir J. Hintikka, Knowledge and Belief...

25. B. Russell, «What is Meant by "A believes p"? ?, p. 159.

26. En revanche, deux énoncés de forme logique $p$ et $q$ correspondent à des faits distincts tout en étant parfaitement compatibles entre eux; l'auteur remercie un des rapporteurs pour avoir apporté cette précision importante. 
leur théorie de la vérité, car celle-ci porte dans les deux cas sur ce qui donne le droit de qualifier un énoncé de vrai ou de faux. Hintikka est lui-même un partisan de la vérité-correspondance, ce qui prouve que sa caractérisation modale de la vérité logique (comme vraie dans tous les mondes possibles) est indépendante de sa théorie de la vérité. Ce n'est donc pas la théorie de la vérité qui distingue les logiques de Russell et Hintikka, puisque tous deux adhèrent à l'idée d'une vérité comme relation entre un langage et des faits. Le différend porte plutôt sur l'usage qui est fait de la notion de vérité en logique modale contemporaine: la vérité n'a plus la proposition (Satz an sich) pour porteur spécifique; un énoncé peut être qualifié lui-même de vrai ou de faux, quelle que soit la nature du fait qu'il exprime; propositions et énoncés sont caractérisés indifféremment en termes de valeurs de vérité, même si leurs conditions de vérité sont distinctes.

Ne risque-t-on pas de perdre de vue le sens primitif de la vérité si celleci désigne indistinctement les conditions de satisfaction d'une formule quelconque, qu'elle soit non modale ou modale? La "vérité dans un modèle» $(v(w, \phi)=1)$ signifie chez Hintikka l'appartenance à un monde possible $w$ (qu'il appelle «ensemble modèle»). Or, si la sémantique relationnelle assimile « appartenance à » un monde et « vérité dans» un monde, ne reproduit-elle pas la confusion des ordres que Russell avait soigneusement évitée par sa distinction entre fonctions de vérité et fonctions de signification? Le problème de fond peut être exprimé ainsi, qui concerne la nature du lien sémantique entre les expressions d'un langage et les faits de la réalité. Selon une formule de Quine,

le prédicat de vérité nous rappelle qu'en dépit d'une montée sémantique qui nous amène à parler des énoncés, notre regard est dirigé vers le monde ${ }^{27}$.

L'est-il encore vraiment en logique modale épistémique, ou ne parle-t-on dans celle-ci de vérité et de fausseté que de façon dévoyée? La question est importante, puisqu'il s'agit de savoir ce qui justifie les conditions de vérité d'un espace épistémique. $\mathrm{Si}$, en effet, on suppose que ce n'est pas une relation de correspondance qui établit les relations possibles entre une croyance et une autre, pour quelle raison faudrait-il encore maintenir le critère de cohérence des logiques modales normales, et quelle raison empêche le logicien de modifier ces règles sémantiques comme bon lui semble?

Le problème est celui de justifier les règles qui déterminent la signification d'une formule modale. Le prétendu lien entre la structure des énoncés d'un

27. W. V. Quine, La philosophie de la logique, J. Largeault (trad.), Paris, Aubier, 1975; édition originale (citée ici): Philosophy of Logic, Englewood Cliffs, Prentice Hall, 1970, p. 24 (traduction française de l'auteur). 
langage et la structure des faits est une hypothèse douteuse, dans la mesure où nous n'aurions jamais accès aux faits eux-mêmes mais seulement à leurs expressions linguistiques. Et si, contrairement à l'approche réaliste de Russell, aucune «structure ontologique» ne garantit les vérités logiques, alors comment les justifier et à quoi sont censées «correspondre » les règles sémantiques dans un espace épistémique? Une solution consiste à traiter les valeurs de vérité comme de simples valeurs algébriques (les symboles 1 et o de la logique booléenne) n'exprimant aucune correspondance avec la réalité extérieure. La porte est alors ouverte au relativisme ou au formalisme en logique. Si le seul critère sémantique restant est l'accord avec nos intuitions de langage, alors la logique n'a plus vocation à corriger nos intuitions ordinaires; mais elle les conforte, au contraire, et une fameuse formule de Russell semble même aller dans ce sens:

Une théorie logique peut être testée selon sa capacité à traiter des énigmes, et c'est un projet salutaire, lorsqu'il s'agit de la logique, que d'emplir l'esprit d'autant d'énigmes que possible, puisque celles-ci servent en grande partie les mêmes besoins que les expérimentations en science physique ${ }^{28}$.

L'usage de la notion de vérité n'est toutefois pas libre, chez Russell, puisque la vérité exprime encore un lien avec la réalité et ne peut pas être redéfinie arbitrairement dans le seul but de résoudre (mieux, de dissoudre) des problèmes d'analyse du langage.

Nous avons mesuré ici le prix à payer pour l'interprétation d'une logique épistémique dans les termes de la logique selon Russell. Une telle logique philosophique pose de sérieuses difficultés en philosophie de la logique, dans la mesure où la notion de vérité doit être examinée avec prudence en ce qui concerne du moins sa relation avec les entités linguistiques et les événements psychologiques. Des résistances similaires rendirent inconcevable le projet d'une logique épistémique chez Wittgenstein ou Quine, notamment, quoique pour des raisons philosophiques quelque peu différentes. Le résultat fut cela dit le même, excluant de fait l'introduction d'un vocabulaire modal au sein de leur logique.

Rien de moins ici que la distinction entre deux façons de pratiquer la logique, selon que l'on considère celle-ci comme un langage universel en prise avec le monde réel ou comme un calcul replié sur ses propres contraintes techniques. L'histoire de la logique a finalement donné raison au formalisme et, rétrospectivement, à la logique symbolique de Hugh

28. B. Russell, «On Denoting», Mind, $\mathrm{n}^{\circ} 14,1905$, p. 479-493, ici p. 484-485. 
MacColl sur son plus grand contempteur que fut Russell. Les obstacles épistémologiques ont fini par être levés et, avec eux, la possibilité de logiques au pluriel. Un monde sépare ainsi la «logique philosophique» telle que Russell l'entendait et celles qui garnissent de nos jours les sections de logique moderne. Nous espérons avoir montré que c'est dans cette philosophie même que se trouve l'origine d'un tel revirement des rapports de force au sein de la discipline logique.

Fabien SCHANG

Université de Maringá (Brésil) 